\title{
Análisis de los factores predictores de amputación de extremidades en pacientes con quemaduras eléctricas de alto voltaje
}

\author{
Guillermo García Álvarez ${ }^{1,3}$, Guillermo Wiegering Cecchi²,3
}

\section{RESUMEN:}

Objetivo: La amputación de extremidades es considerada una de las consecuencias más devastadoras de la injuria eléctrica. Cualquier factor que se correlacione con el grado de daño muscular se puede utilizar para predecir la necesidad de amputación de extremidades. El objetivo de este estudio fue determinar los factores que pueden ser utilizados para predecir la amputación de extremidades en pacientes con quemaduras eléctricas de alto voltaje.

Material y Métodos: Noventa y siete pacientes con quemaduras eléctricas fueron ingresados al Servicio de Cirugía Plástica, Reconstructiva y Quemados del Hospital Nacional Arzobispo Loayza durante un período de 5 años. Se realizó un análisis retrospectivo de los posibles factores de riesgo asociados entre los pacientes en los cuales se realizó una amputación y aquellos que no.

Resultados: Un total de 64 pacientes fueron incluidos para el análisis. Quince pacientes fueron sometidos a amputaciones de extremidades. El análisis multivariado de los factores de riesgo entre los grupos de amputación y no amputación mostró significación estadística para los niveles de la isoenzima CPK-MB en las primeras 24 horas. Un nivel de CPK-MB sérico por encima de 14,955 U/L predijo alto riesgo de amputación de extremidades con alta especificidad (83\%) y sensibilidad (76\%). Sólo un paciente con una notable disminución en los niveles de CPKt y CPK-MB tras fasciotomía evitó una amputación mayor de la extremidad.

Conclusiones: Nuestros resultados sugieren que el nivel de CPK-MB es un factor independiente de predicción de amputación de extremidades en paciente con quemaduras eléctricas de alto voltaje. Sugerimos que la adición de la isoenzima CPK-MB como parámetro laboratorial puede ser un método valioso para la detección precoz de daño muscular. (Horiz Med 2015; 15(3): 13-19)

Palabras clave: Creatina fosfoquinasa, creatina fosfoquinasa isoenzima MB, amputación de extremidad, quemadura eléctrica de alto voltaje. (Fuente: DeCS BIREME)

\section{Analysis of predictor factors of limb amputation in patients with high-voltage electrical burns}

\section{ABSTRACT}

Objective: Limb amputation is considered one of the most devastating consequences of electrical injury. Any factors that correlate with the degree of muscle damage can be used to predict the necessity of limb amputation. The aim of this study was to determine the factors that can be used to predict limb amputation in high-voltage electrically injured patients. Material and Methods: Ninety-seven high-voltage electrically injured patients were admitted to the Department of Plastic and Reconstructive Surgery and Burns of Hospital Nacional Arzobispo Loayza on a 5 year period. A retrospective analysis of the possible related risk factors between amputation and non-amputation patients was performed.

Results: A total of 64 patients were enrolled for analysis. Fifteen patients underwent limb amputations. Multivariate analysis of the risk factors between amputation and non-amputation groups showed statistical significance for first 24 hour creatine kinase-isoenzyme MB (CKMB) level. A serum CK-MB level above 14,955 U/L predicted high risk of limb amputation with high specificity $(83 \%)$ and sensitivity $(76 \%)$. Only one patient with a remarkable decrease of creatine kinase (CPKt) and CK-MB levels after fasciotomy avoided a major limb amputation.

Conclusions: Our results suggest that CPK-MB level is an independent factor for prediction of limb amputationin patients with high-voltage electrical burns. We suggest that the addition of CPK-MB evaluation to clinical symptom screening may be a valuable method for early detection of muscle damage. (Horiz Med 2015; 15(3): 13-19)

Key words: Creatine kinase, creatine kinase isoenzyme MB, limb amputation, high voltage electrical injury. (source: MeSH NLM)

\footnotetext{
Médico Residente de la Especialidad de Cirugía Plástica y Reconstructiva.

Cirujano Plástico. Médico Asistente del Servicio de Cirugía Plástica y Reconstructiva.

Servicio de Cirugía Plástica, Reconstructiva y Quemados del Hospital Nacional Arzobispo Loayza, Lima-Perú.
} 


\section{INTRODUCCIÓN}

La injuria eléctrica es una de las formas más graves de lesiones en la población civil en la sociedad industrializada. Aunque la tasa de mortalidad se ha reducido considerablemente debido a las mejoras en la reanimación y cuidados intensivos, la alta morbilidad, especialmente la amputación de extremidades, perjudica la capacidad funcional de estos pacientes, cambiando radicalmente su estilo de vida posterior a una lesión eléctrica de alta tensión $(1,2)$.

A diferencia de las quemaduras térmicas por fuego directo o líquido caliente en las cuales la extensión de la lesión es evidente a simple vista; las quemaduras eléctricas pertenecen a un tipo especial de quemaduras, en donde, si bien es posible evidenciar las lesiones que produce la corriente eléctrica superficialmente, existen lesiones profundas u "ocultas" graves, sobretodo de tejido muscular, cubiertas por piel sana, que sufren una "necrosis progresiva" con el paso de los días e incluso semanas, que puede conllevar a la realización de una amputación en estos pacientes (3). La tasa de amputación reportada en la literatura varía de un $10 \%$ a un $68 \%$ (4).

La teoría del calentamiento eléctrico de Joule ha sido incluida dentro de la fisiopatología principal del daño tisular en lesiones eléctricas (5). Pero en las últimas décadas, se ha observado que se puede producir un daño no-térmico en los tejidos, cuando la corriente eléctrica provoca alteraciones en la membrana celular, que se conoce como electroporación y electroconformación, que puede contribuir a la necrosis muscular y posterior amputación de extremidades $(6,7)$.

La evaluación de la viabilidad del tejido en lesiones eléctricas de alto voltaje debe llevarse a cabo tan pronto como sea posible para la prevención de la amputación de extremidades.

La gammagrafía con pirofosfato de tecnecio-99m (99mTc-PEP) (8) y la resonancia magnética nuclear RMN (9) son procedimientos para medir el área de necrosis muscular y el cambio morfológico de los vasos sanguíneos antes de tomar una decisión definitiva de amputación.
Sin embargo, estos métodos no deben ser la única evidencia que justifique e indique la amputación de extremidades.

Se ha propuesto que la elevación de los niveles de las enzimas musculares puede ser utilizada para determinar el daño muscular en pacientes con quemaduras eléctricas de alto voltaje. Hay evidencia que un alto nivel sérico de creatina fosfoquinasa (CPK) se correlaciona estrechamente con una alta tasa de amputación $(10,11)$.

Cualquiera de los parámetros de laboratorio, como son las enzimas tisulares, que se correlacionen directamente con la extensión del daño muscular son valiosos para poder detectar y catalogar a estos pacientes con un alto riesgo de amputación de extremidades, orientando al cirujano y apoyando la decisión de una descompresión quirúrgica temprana de las extremidades y un manejo agresivo en este tipo de pacientes.

El propósito de este estudio fué evaluar retrospectivamente la data de los pacientes eléctricamente lesionados mediante un análisis multivariado para revelar los factores que contribuyen a la amputación de extremidades.

\section{MATERIAL Y MÉTODOS}

Del año 2010 al 2014, 97 pacientes con lesión eléctrica de alto voltaje ( $>1000 \mathrm{~V}$ ) fueron ingresados al Servicio de Cirugía Plástica, Reconstructiva y Quemados del Hospital Nacional Arzobispo Loayza, de los cuales 64 pacientes fueron incluidos para el análisis. Se llevó a cabo una revisión retrospectiva de los factores que influyen en la amputación de extremidades en pacientes con quemaduras eléctricas de alto voltaje, que incluyó los siguientes factores: edad, superficie corporal quemada (SCQ\%), presencia de síndrome compartimental, diabetes, mioglobulinuria y otra lesión asociada, así mismo se realizaron dosajes séricos de CPKt (Creatina fosfoquinasa total) dentro y después de las primeras 24 horas de producida la injuria y CPK$M B$ (creatina fosfoquinasa isoenzima MB) dentro y después de las primeras 24 horas de producida la injuria eléctrica. 
Se realizó fasciotomía inmediata de las extremidades en pacientes con presentación clínica de síndrome compartimental, como compartimentos tensos e hinchados, parestesias y dolor estiramiento pasivo. Los pacientes que fallecieron al ingreso de la emergencia o presentaban lesión miocárdica (arritmias, cambios ST-T o CK-MB relación quinasa creatina por encima del 5\%), o que ingresaron al hospital después de las 24 horas de ocurrido el accidente fueron excluidos del estudio.

Se utilizó la prueba exacta de Fisher para comparar los datos categóricos entre los grupos de amputación y no amputación; se utilizaron pruebas t para analizar la diferencia entre dos variables continuas. Se realizó una regresión logística para el análisis multivariado de los posibles factores relacionados.

Para predecir la ocurrencia de amputación, se aplicó una curva ROC con el software MedCalc (versión 18.0) para determinar el punto de corte de las concentraciones séricas de CPKt y CPK-MB. El análisis estadístico se realizó con el programa SPSS versión 21.0. Los valores de $p<0,05$ fueron considerados estadísticamente significativos. Los datos se expresaron como media \pm DS (desviación estándar).

\section{RESULTADOS}

Un total de 64 pacientes fueron incluidos para el análisis. Todos los pacientes fueron de sexo masculino, la edad media fue de $32 \pm 12$ años, y la media de la SCQ fue de $25 \pm 18 \%$.

A 15 pacientes se les realizó amputación de extremidades, de los cuales 8 pacientes fueron sometidos a amputaciones digitales $(10,1 \%$ ) y 7 fueron sometidos a una amputación mayor de extremidad $(8,7 \%)$. Cuatro pacientes fueron sometidos a una fasciotomía inmediata por síndrome compartimental.

Se realizó el análisis univariado y multivariado para evaluar los efectos independientes de los factores de riesgo de amputación en pacientes afectados eléctricamente.

El análisis univariado de los factores de riesgo entre los grupos de amputación y no-amputación mostró significación estadística para la presencia de síndrome compartimental y niveles séricos de CPK-MB en las primeras 24 horas de producida la injuria (Tabla 1).

Tabla 1. Análisis univariado de los factores de riesgo entre los grupos de amputación y no-amputación.

\begin{tabular}{|c|c|c|c|}
\hline & Amputación (n=13) & Sin Amputación ( $n=55)$ & $\mathbf{p}$ \\
\hline Edad & $30 \pm 9$ & $32 \pm 11$ & 0.619 \\
\hline $\operatorname{SCQ}(\%)$ & $10.0 \pm 8.8$ & $15.3 \pm 10.9$ & 0.457 \\
\hline CPKt (U/L) <24h & $19.707 \pm 11.550$ & $13.786 \pm 6.160$ & 0.058 \\
\hline CPKt $(\mathrm{U} / \mathrm{L})>24 \mathrm{~h}$ & $13.098 \pm 10.440$ & $9.151 \pm 7.122$ & 0.061 \\
\hline CPK-MB (U/L) <24h & $14.495 \pm 235$ & $625 \pm 131$ & $0.006^{*}$ \\
\hline CPK-MB (U/L) $>24 h$ & $10.458 \pm 210$ & $9.478 \pm 128$ & 0.170 \\
\hline DHL (U/L) & $637 \pm 308$ & $522 \pm 205$ & \\
\hline \multicolumn{4}{|c|}{ Síndrome compartimental } \\
\hline Si & 4 & 0 & $0.009^{*}$ \\
\hline No & 9 & 55 & \\
\hline \multicolumn{4}{|l|}{ Injuria asociada } \\
\hline $\mathrm{Si}$ & 4 & 16 & 0.560 \\
\hline No & 9 & 39 & \\
\hline \multicolumn{4}{|l|}{ Diabetes } \\
\hline $\mathrm{Si}$ & 0 & 1 & 0.812 \\
\hline No & 13 & 54 & \\
\hline \multicolumn{4}{|l|}{ Mioglobulinuria } \\
\hline Si & 5 & 15 & 0.302 \\
\hline No & 8 & 40 & \\
\hline
\end{tabular}




\section{Curva ROC}

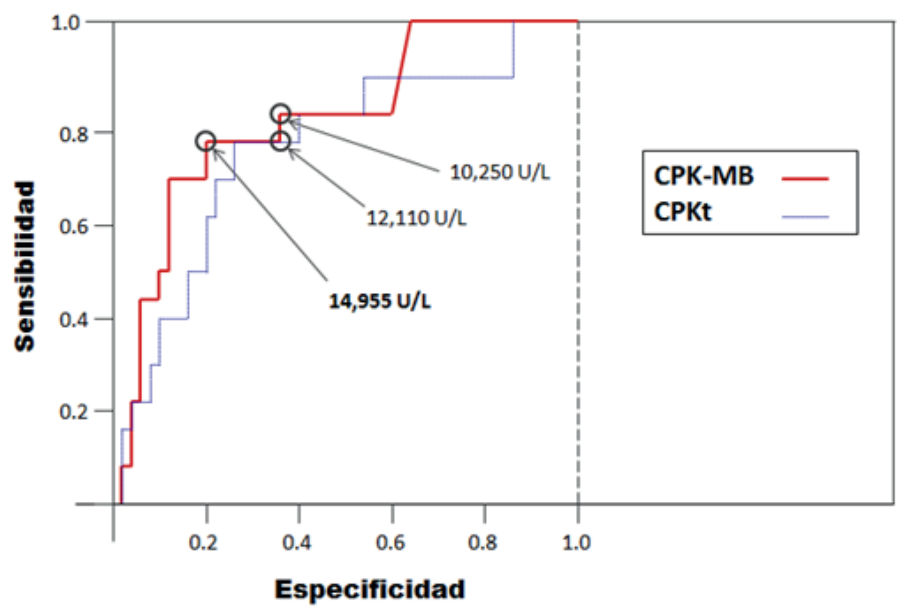

Figura 1. Diagrama de curvas ROC (Receiver Operating Characteristic), comparando los niveles en suero de la CPK-MB (línea roja) y la CPKt (línea azul) en pacientes con quemaduras eléctricas de alto voltaje. Se marcan los puntos de corte de los valores séricos de CPK-MB en 10,250 U/L; 12,110 U/L y 14,955 U/L. Las áreas bajo la curva de CPK-MB y CPKt son 0.879 y 0.772 , respectivamente.
Para evaluar si los niveles séricos de CPKt y CPKMB podrían predecir la amputación de miembros, se trazó una curva ROC para evaluar el poder discriminatorio de la prueba. La sensibilidad y la especificidad de CPKt y CPK-MB como prueba de detección se analizaron en el rango de 890-175,650
U/L para CPKt y de 396 a 80,335 U/L para CPK-MB. Los puntos de corte se representaron para generar una curva.

El área bajo la curva ROC es mayor para CPKMB $(0,879)$ que para CPKt $(0,772$, Figura 1$)$. El

Tabla 2. Valores y coordenadas de la curva ROC para CPK-MB.

\begin{tabular}{|c|c|c|c|c|}
\hline CPK - MB (U/L) & Sensibilidad & IC 95\% & Especificidad & IC $95 \%$ \\
\hline$>396$ & 100.00 & $75.3-100.0$ & 0.00 & $00-6.4$ \\
\hline$>3.578$ & 100.00 & $75.3-100.0$ & 39.29 & $26.5-53.2$ \\
\hline$>6.700$ & 92.31 & $64.0-99.8$ & 41.07 & $28.1-55.0$ \\
\hline$>8.147$ & 84.62 & $54.6-98.1$ & 42.86 & $29.7-56.8$ \\
\hline$>10.250^{*}$ & 84.62 & $54.6-98.1$ & 67.86 & $54.0-79.7$ \\
\hline$>12.110^{*}$ & 76.92 & 46.2 - 95.0 & 67.86 & $54.0-79.7$ \\
\hline$>14.955^{\star}$ & 76.92 & $46.2-95.0$ & 83.93 & $71.7-92.4^{*}$ \\
\hline$>15.894^{*}$ & 69.23 & $38.6-90.0$ & 83.93 & $71.7-92.4$ \\
\hline$>18.875$ & 69.23 & $38.6-90.0$ & 89.29 & $78.1-96.0$ \\
\hline$>25.743$ & 53.85 & $25.1-80.8$ & 89.29 & $78.1-96.0$ \\
\hline$>28.700$ & 53.85 & $25.1-80.8$ & 91.07 & $80.4-97.0$ \\
\hline$>29.184$ & 46.15 & $19.2-74.9$ & 91.07 & $80.4-97.0$ \\
\hline$>31.745$ & 46.15 & $19.2-74.9$ & 94.64 & $85.1-98.9$ \\
\hline$>32.223$ & 23.08 & $5.0-53.8$ & 94.64 & $85.1-98.9$ \\
\hline$>45.874$ & 23.08 & $5.0-53.8$ & 96.43 & $87.7-99.6$ \\
\hline$>50.689$ & 7.69 & $0.2-36.0$ & 96.43 & $87.7-99.6$ \\
\hline$>59.290$ & 7.69 & $0.2-36.0$ & 98.21 & $90.4-100.0$ \\
\hline$>65.284$ & 0.00 & $0.0-24.7$ & 98.21 & $90.4-100.0$ \\
\hline$>80.335$ & 0.00 & $0.0-24.7$ & 100.00 & $93.6-100.0$ \\
\hline
\end{tabular}


valor umbral de $14,955 \mathrm{U} / \mathrm{L}$ produjo una misma sensibilidad de $77 \%$, pero una mejor especificidad (84\% vs $68 \%$ ) con el valor de $12,110 \mathrm{U} / \mathrm{L}$.

Además, el valor umbral de $10,250 \mathrm{U} / \mathrm{L}$ produjo una mayor sensibilidad de $84 \%$, pero una menor especificidad de $67 \%$ (Tabla 2). Por lo tanto, decidimos que el mejor valor de corte para la CPKMB fue de 14,955 U/L.

El nivel de CPKt se fijó en 19,233 U/L (sensibilidad: $78 \%$, especificidad: $76 \%$ ). Para expresar la relación entre los pacientes con un nivel de CPKt por encima de 19,256 U/L y los que tienen un nivel por debajo de $19,256 \mathrm{U} / \mathrm{L}$, se obtuvo un OR (Odds ratio) en 10,0 (95\% intervalo de confianza: $2,4-41,6)$, o sea hay 10 veces mayor riesgo de sufrir de amputación con un nivel de CPKt por encima de 19,256 U/L.
Por el contrario, se obtuvo un OR (Odds ratio) de 18, 5 (95\% intervalo de confianza: $5,0-77,0)$ en pacientes con niveles séricos de CPK-MB por encima de 14,955 $U / L$, lo que indica un mejor valor predictivo del CPK-MB con respecto al CPKt, en el riesgo de sufrir una amputación de extremidad en pacientes con quemaduras eléctricas de alto voltaje.

El único factor que alcanzó significación estadística en el análisis univariado fué:

La concentración sérica en CPK-MB.

Tabla 3. Regresión logística multivariada, análisis de los factores de riesgo de amputación

\begin{tabular}{|c|c|c|c|}
\hline & Amputación (n=13) & No Amputación $(n=55)$ & p \\
\hline \multicolumn{4}{|c|}{ CPKt $<24 \mathrm{~h}(\mathrm{U} / \mathrm{L})$} \\
\hline$<19.300$ & 4 & 46 & \multirow[b]{2}{*}{0.552} \\
\hline$>19.300$ & 9 & 9 & \\
\hline \multicolumn{4}{|c|}{ CPKt $-M B<24 h(U / L)$} \\
\hline$<14.955$ & 2 & 51 & \multirow[b]{2}{*}{$0.00001^{*}$} \\
\hline$>14.955$ & 11 & 4 & \\
\hline \multicolumn{4}{|c|}{ Fasciotomía } \\
\hline Si & 4 & 0 & \multirow[b]{2}{*}{0.157} \\
\hline No & 9 & 55 & \\
\hline
\end{tabular}

\section{DISCUSIÓN}

Las lesiones eléctricas causan daño muscular y por consiguiente, elevación marcada de enzimas musculares. La CPKt y la isoenzima CPK-MB se detectan fácilmente en la circulación y reflejan lesión muscular importante.

Los niveles altos de creatina fosfoquinasa se correlacionan con el riesgo de amputación de extremidades después de una injuria eléctrica $(10,11)$. La creatina fosfoquinasa tiene tres isoformas: $M M, M B, y B B$, con una amplia distribución en tejidos tales como músculo esquelético, músculo cardíaco, el hígado, los eritrocitos, cerebro y músculo liso (12).

Cuando la corriente eléctrica pasa a través de estos tejidos, las isoformas variables de CPK se liberan en el torrente sanguíneo y en consecuencia se produce una elevación de creatina fosfoquinasa total (CPKt).

Sin embargo, esta elevación no refleja el verdadero daño a nivel del músculo esquelético. Esto puede explicar por qué nuestra CPKt no tenía ninguna asociación significativa en la predicción del riesgo 
de amputación de extremidades en nuestro análisis. Aproximadamente el $99 \%$ de la CPK total en el músculo esquelético es CPK-MM (13), y el aumento del nivel de CPK-MM se correlaciona de la misma forma que la CPK total, la cual no tiene ninguna asociación significativa en la predicción de riesgo de amputación de extremidades.

Por el contrario, se sabe que la CPK-MB tiene un origen miocárdico y es un predictor de lesión miocárdica (14).

La lesión del músculo esquelético también puede liberar grandes cantidades de CPK-MB, sin embargo para descartar la posibilidad de que se tratara de una afección cardíaca, la proporción de CPK-MB del CPKt, debe ser inferior al 5\% (15).

Una enfermedad o daño del músculo esquelético; en este caso, una injuria eléctrica, es más probable, si el porcentaje de CPK-MB es menor al $5 \%$ con respecto al CPKt, porque se ha demostrado que en el suero de pacientes con infarto agudo de miocardio en las primeras $48 \mathrm{~h}$ después del inicio de los síntomas, los niveles de CPK-MB en la mayoría de los casos son mayores al 5\% (entre $5.1 \%$ y $23.6 \%$ de la CPKt) (15).

En nuestro estudio las proporciones, en los grupos de amputación y no-amputación, fueron menores al $5 \%$, excluyendo la posibilidad de un CPK-MB elevado, debido a una lesión miocárdica subclínica.

Además, el hallazgo en nuestro estudio sugiere que el CPK-MB en las primeras 24 horas, es un predictor de pronóstico para el resultado clínico de las extremidades lesionadas.

La misma conclusión sobre el origen de la CPK-MB de músculo esquelético también se encontró en los casos tanto de traumatismos músculo esquelético y lesiones eléctricas $(16,17)$.

El valor umbral de 14,955 U/L de CPK-MB sérico encaja con el mejor equilibrio entre la especificidad y la sensibilidad en nuestro estudio. Sin embargo, si se hubiera utilizado el valor de $12,110 \mathrm{U} / \mathrm{L}$, el $34 \%$ de los pacientes en el grupo de no-amputación habrían sido falsos positivos, en comparación con
$15,3 \%$ de resultados falsos positivos al utilizar el valor de corte de si 14,955 U/L.

Por otra parte, el nivel de CPK-MB en 14,955 U/L se asoció significativamente con la amputación de extremidades en el análisis multivariado. Por 10 tanto, tenemos 14,955 U/L como nuestro mejor punto de corte para predecir la amputación de extremidades en pacientes con quemaduras eléctricas de alto voltaje.

La posibilidad de descompresión inadecuada tras fasciotomía que lleva a la amputación debe ser considerada. Sin embargo, se necesitan más casos para dilucidar los valores. Aunque el riesgo de amputación en nuestros resultados se incrementa de forma independiente con el nivel inicial de la CPK-MB en suero, la utilidad de un único factor para predecir la amputación debe considerarse con mucho criterio.

La RMN de alta resolución puede ser una herramienta útil para la detección precoz de la necrosis muscular profunda (18). La fasciotomía selectiva, sin embargo, con base en los hallazgos clínicos como compromiso neurovascular y aumento de la presión del compartimiento está generalmente aceptada (4).

El valor de la isoenzima CPK-MB en nuestro estudio es más importante que la CPKt para predecir daño muscular, consistente con un estudio previo (18). Por lo tanto, sugerimos que la adición de la CPKMB y la evaluación de los síntomas clínicos son un valioso y conveniente método para la detección temprana del daño muscular y la prevención de la lesión muscular progresiva y finalmente la amputación de extremidades en pacientes con quemaduras eléctricas de alto voltaje.

En conclusión, este estudio muestra que altos niveles en suero de CPK-MB en las primeras 24 horas de ocurrida la injuria eléctrica, se correlaciona estrechamente con la amputación de extremidades en pacientes con quemaduras de alto voltaje.

Se encontró que los pacientes con un nivel sérico de CPK-MB por encima de 14,955 U/L, tienen un mayor riesgo de amputación que aquellos con niveles por debajo de 14,955 U/L. 
Fuentes de financiamiento

Este artículo ha sido financiado por los autores.

\section{Conflicto de interés}

Los autores declaran no tener ningún conflicto de interés.

\section{Correspondencia:}

\author{
Guillermo García Álvarez \\ Dirección: Clínica Javier Prado. \\ Av. Javier Prado Este 499 Of. 215. San Isidro Perú. \\ Teléfono: + (51 1) 940248747 \\ Correo electrónico: antongar77@hotmail.com
}

\section{REFERENCIAS BIBLIOGRÁFICAS}

1. Arnoldo BD, Purdue GF, Kowalske K, Helm PA, Burris A, Hunt JL. Electrical injuries: a 20-year review. J Burn Care Rehabil 2004; 25:479-84.

2. Maghsoudi $\mathrm{H}$, Adyani $\mathrm{Y}$, Ahmadian N. Electrical and lightning injuries. J Burn Care Res 2007;28:255-61.

3. Bolgiani A, Lima E, Freitas M. Quemaduras, conductas clínicas y quirúrgicas. 1er Rev. Wiegering G. Sao Paulo, Atheneu, 2013; p:4952.

4. Arnoldo B, Klein M, Gibran NS. Practice guidelines for the management of electrical injuries. J Burn Care Res 2006;27:439-47.

5. Lee RC, Kolodney MS. Electrical injury mechanisms: dynamics of the thermal response. Plast Reconstr Surg 1987; 80:663-71.

6. Block TA, Aarsvold JN, Matthews 2nd KL, Mintzer RA, River LP, Capelli-Schellpfeffer M, et al. The 1995 Lindberg Award. Nonthermally mediated muscle injury and necrosis in electrical trauma. J Burn Care Rehabil 1995; 16:581-8.

7. Lee RC, Astumian RD. The physicochemical basis for thermal and non-thermal 'burn' injuries. Burns 1996; 22:509-19.

8. Hunt J, Lewis S, Parkey R, Baxter C. The use of Technetium-99m stannous pyrophosphate scintigraphy to identify muscle damage in acute electric burns. J Trauma 1979; 19:409-13.

9. Chen YX, Xu Y, Guo ZR, Chai JK, Hu XJ, Zhang ZM, et al. The application of ultrasonography in the diagnosis of deep electric injury. Zhonghua Shao Shang Za Zhi 2003; 19:38-41.

10. Ahrenholz DH, Schubert W, Solem LD. Creatine kinase as a prognostic indicator in electrical injury. Surgery 1988; 104:741-7.
11. Kopp J, Loos B, Spilker G, Horch RE. Correlation between serum creatinine kinase levels and extent of muscle damage in electrical burns. Burns 2004; 30:680-3.

12. Takagi Y, Yasuhara T, Gomi K. Creatine kinase and its isozymes. Rinsho Byori 2001; Suppl. 116:52-61.

13. Fish RM, Geddes LA, Babbs CF. Medical and bioengineering aspects of electrical injuries. Arizona: Lawyers and Judges Publishing Company; 2003.

14. Chandra NC, Siu CO, Munster AM. Clinical predictors of myocardial damage after high voltage electrical injury. Crit Care Med 1990; 18:293-7.

15. Prellwitz W, Kapp S, Neumeier D, Knedel M, Lang H, Heuwinkel D. Isoenzymes of creatine kinase: distribution in the skeletal muscle and in sera of patients with muscular diseases or damages. Klin Wochenschr 1978; 56:559-65.

16. MC Bride JW, Labrosse KR, Mc Coy HG, Ahrenholz DH, Solem LD, Goldenberg IF. Is serum creatine kinase-MB in electrically injured patients predictive of myocardial injury?. JAMA 1986; 255:764-8.

17. Schwartz JG, Prihoda TJ, Stuckey JH, Gage CL, Darnell ML. Creatine kinase $M B$ in cases of skeletal muscle trauma. Clin Chem 1988; 34:898-901.

18. Pereira C, Fram R, Herndon D. Serum creatinine kinase levels for diagnosing muscle damage in electrical burns. Burns 2005; 31:670-1.
Recibido: 21 de Junio de 2015 Aprobado: 06 de Agosto de 2015 